\title{
Jahresbericht 2006 der Arbeitsgemeinschaft der Ethikkommissionen für klinische Versuche AGEK
}

\section{Dr. Georg Kreienbühl}

Präsident AGEK, St. Gallen

Korrespondenz:

Geschäftsstelle AGEK

Prof. Dr. Niklaus Tüller

Postfach 514

CH-3000 Bern 8

Tel. 0313112988

niklaus.tueller@swissonline.ch
An der Versammlung vom 23. November 2005 wurden die Statuten der AGEK nach intensiven Vorarbeiten genehmigt. Dieses Datum kann also als Gründungstag der AGEK bezeichnet werden. Wichtige Impulse kamen dabei von der SAMW, insbesondere von Hermann Amstad und von Anton Nanzer, auf dessen Vorschlag hin wir Herrn alt Bundesrichter Heinz Hausheer beigezogen haben, der uns eine grosse Hilfe war.

Im Folgenden gebe ich einen Überblick über die wichtigsten Problemfelder, die wir behandelt haben.

\section{Versicherungen}

Auf Initiative unserer Tessiner Kollegen, insbesondere von Stefano Radczuweit, fand betr. Versicherung und Versicherungszertifikat zuerst eine Aussprache zwischen Herrn Schneller und Herrn Dietschy (Swissmedic) einerseits und Niklaus Tüller und dem Präsidenten andererseits statt. An insgesamt drei Sitzungen mit Vertretern der Swissmedic, SGCI (Schweizerische Gesellschaft für Chemische Industrie), der Versicherungswirtschaft und der AGEK konnten wir uns auf zwei Dokumente einigen, nämlich «Anforderungen an eine Versicherung für klinische Versuche mit Heilmitteln am Menschen» und ein «Versicherungszertifikat zuhanden der schweizerischen Ethikkommissionen». Formal war dies ein Erfolg, indem nun Swissmedic und unsere Website «Swissethics» die gleichen Dokumente aufgeschaltet haben. Das Vorgehen wurde an der AGEK-Sitzung vom 24. August 2005 einstimmig in Abwesenheit der opponierenden Tessiner Kollegen gebilligt. Die Tessiner Kollegen opponierten gegen den nachfolgenden Ausschlussgrund:

\begin{abstract}
«Personenschäden einer Versuchsperson, die nicht über ein nach dem Stand der medizinischen Wissenschaften zu erwartendes Mass an unerwünschten Wirkungen des zu prüfenden Heilmittels hinausgehen.»
\end{abstract}

Am 29./30. September 2005 fand in Zürich eine von den Professoren Sprumont und Poledna organisierte Tagung zum Thema «Klinische Versuche: Art und Umfang der Schadensdeckung für die
Versuchspersonen» statt. Die AGEK wurde offiziell zu dieser Tagung eingeladen. Dabei zeigte insbesondere das Referat von Christoph Zenger klar, dass die obenzitierte Einschränkung nicht zulässig ist. Sie setzt die Ethikkommissionen einem Risiko aus. Die Opposition unserer Tessiner Kollegen ist also mehr als berechtigt. Auf Initiative der SGCI fand am 28. April 2006 eine Aussprache interessierter Kreise (Industrie, Oncosuisse, Versicherungswirtschaft und AGEK) zu diesem Problem statt, an der Christoph Zenger und der Präsident teilnahmen. Wir sind optimistisch, dass hier Fortschritte erzielt werden.

\section{Motion Dunant}

Vereinfacht gesagt verlangt die Motion Dunant eine Zusammenlegung der Ethikkommissionen. Niklaus Tüller und der Präsident hatten die Möglichkeit, nach der Behandlung im Nationalrat mit Herrn Prof. Gutzwiller zu sprechen. Zur Sitzung der Kommission Wissenschaft, Bildung und Kultur des Ständerates wurden wir (die Geschäftsleitung, also Kreienbühl, Tüller, Burnier) auf unser Ersuchen hin am 30. Januar 2006 eingeladen und konnten unsere Sichtweise darlegen. Die AGEK hatte dazu ein Argumentarium erarbeitet. Trotzdem wurde die Motion in der Kommission mit 6:4 Stimmen angenommen. Auch der Ständerat stimmte mit Stichentscheid des Präsidenten zu.

Wir können davon ausgehen, dass die Motion keine direkten Wirkungen haben wird, aber im Rahmen der Behandlung des Humanforschungsgesetzes HFG in den Räten abgeschrieben werden wird.

\section{Humanforschungsgesetz HFG}

Im Rahmen der Vernehmlassung zum Humanforschungsgesetz hat auch die AGEK Stellung genommen. Das Referat von Prof. Doppelfeld an der Sitzung vom November 2005 erwies sich dabei als guter Einstieg zum Thema. Erfreulicherweise stellte sich Dr. Michael Gerber, Chef des Rechtsdienstes des federführenden BAG, für ein Referat zur Verfügung (17. März 2006). Die Stellungnahme der AGEK wurde an der Sitzung vom 
19. April 2006 erarbeitet. Unsere Stellungnahme wurde redaktionell von Jürg Vontobel formuliert. Nach unseren Informationen wird das Resultat der Vernehmlassung erst nächstes Jahr bekanntwerden.

\section{Aus- und Weiterbildung von EK-Mitgliedern}

Gemäss Art. 29 Ziffer 3 gilt:

Sie (d.h. die Kantone) sorgen für die Aus- und Weiterbildung der Mitglieder der Ethikkommissionen. Das Institut (d.h. Swissmedic) unterstützt die Kantone dabei und versorgt die Mitglieder der Ethikkommissionen mit Fachinformation.

Bisher wurde die Fortbildung von der Schweizerischen Gesellschaft für Biomedizinische Ethik und der Schweizerischen Akademie der Medizinischen Wissenschaften gemeinsam organisiert. Für diese Pionierleistung gebührt beiden Organisationen unser Dank.

Die bisherige Form der Weiterbildung vermochte aber nicht in allen Punkten zu befriedigen. In Zusammenarbeit mit KoBeK konnte die Weiterbildung auf eine neue Grundlage gestellt werden. Die 2007 erstmals angebotenen Kurse werden von Christoph Zenger, Leiter der Nachdiplomstudiengänge «Management im Gesundheitswesen» der Universität Bern, und von Dominique Sprumont, Institut de droit de la santé, Université de Neuchâtel, geleitet. Wir sind stolz darauf, dass zwei hervorragend ausgewiesene Fachleute für diese Aufgabe gewonnen wurden.

Für die Zukunft müssen sicher ein Obligatorium der Weiter- und Fortbildung für Ethikkommissionsmitglieder und eine Zertifizierung der Kurse ins Auge gefasst werden. Zuerst wollen wir jetzt aber mit dieser neuen Form Erfahrungen sammeln. Die AGEK hat die Möglichkeit, Anregungen für den Inhalt und die Verbesserung dieser Kurse einzubringen.

\section{KoBeK (Interdisziplinäre Arbeits- gruppe «Koordination der Beurteilung klinischer Versuche»)}

In der KoBeK sind die SAMW, das BAG, die GDK, Swissmedic, die Kantonsapotheker und die AGEK vertreten. Sie hat seit ihrer Gründung im Jahr 2005 als Nachfolgeorganisation der AG StaR sechs Sitzungen durchgeführt. Sie ist ein sinnvolles und wichtiges Instrument, um Probleme interdisziplinär besprechen zu können. Wir möchten an dieser Stelle dem Präsidenten Dominique Sprumont und dem Sekretär Hermann Amstad für den Einsatz herzlich danken. Im Folgenden liste ich eine Auswahl der bearbeiteten Themen auf:

- Weiter- und Fortbildung von EK-Mitgliedern;

- Praxiserfahrungsberichte PEB;

- Probanden- und Studienregister;

- Forschung an Urteilsunfähigen und in Notfallsituationen (siehe «Forschung in Notfallsituationen und mit Personen, welche vorübergehend oder dauernd urteilsunfähig sind», abrufbar unter www.swissethics.ch $\rightarrow$ Checklisten);

- Motion Dunant und HFG;

- Zusammenarbeit mit dem und Formulare des Nationalfonds (SNF);

- Meldungen von Serious Adverse Events (SAE).

\section{Swissmedic}

Robert Kenzelmann hat die Industrie und die AGEK eingeladen, an einem Entwurf einer Verwaltungsverordnung zu den Praxiserfahrungsberichten mitzuarbeiten. Die Arbeiten sind weit fortgeschritten, und der Entwurf dürfte bald in die Vernehmlassung gehen. Zudem haben erste von Swissmedic initiierte Gespräche mit interessierten Kreisen zum Thema, wieweit Swissmedic ein eigenes, öffentlich zugängliches Studienregister führen soll, stattgefunden.

In einem fruchtbaren Gespräch haben wir mit Swissmedic (Robert Kenzelmann) die Probleme der Zusammenarbeit Ethikkommissionen-Swissmedic besprochen.

Wir wurden zudem an einen Informationsaustausch zwischen Swissmedic und ihren Klienten zum Thema Prozessanalyse eingeladen. Diese Prozessanalyse hat ja bekanntlich zu den tiefgreifenden personellen Änderungen geführt, die Ihnen aus der Presse bekannt sind.

\section{Zusammenarbeit mit der SAMW}

Wie aus dem oben Ausgeführten hervorgeht, ist die Zusammenarbeit mit der SAMW sehr fruchtbar. Im Januar 2006 wurde wieder die traditionelle Tagung mit Repräsentanten aus dem Bereich «Klinische Versuche» durchgeführt. Hauptthema war die Ausbildung von Prüfärzten und EK-Mitgliedern in GCP (Good Clinical Practice). Die Tradition dieser Veranstaltung möchten wir unbedingt fortführen.

\section{Dank}

Mein Dank geht an die Mitglieder der Geschäftsleitung, insbesondere an Niklaus Tüller für eine freundschaftliche und kollegiale Zusammenarbeit. Besonders bedanken möchte ich mich bei folgenden Juristen der KEK Bern, die uns immer wieder mit Rat, Tat und Zeit zu Hilfe kamen: Thomas Schuler, Jürg Vontobel, Christoph Zenger. 